DE DE GRUYTER OPEN

\title{
ASSESSMENT OF THE MECHANICAL PROPERTIES OF PET POLYMER MATERIAL FROM RECOVERED PLASTIC BOTTLES

\author{
Irene Villafañe ${ }^{1}$, Colin Keogh ${ }^{2}$, Thomas P. Curran', \\ Emmanuel G. Reynaud ${ }^{3}$
}

Key words: Polymer, PET, Waste Management, Recycling, Mechanical Testing

\begin{abstract}
As the production of single use plastic packaging materials is increasing rapidly, the resultant generation of waste material is quickly becoming one of the world's major environmental issues. These plastic materials are highly stable, with excellent material properties which can allow them to be used beyond their intended single use. To effectively utilize these recovered plastic materials, an understanding of their mechanical properties is required. The aim of this study was to assess the mechanical properties of recovered plastic bottles to prove the feasibility of utilisation for additional uses in non-shredding based recycling systems with lower energy requirements. This system would conserve the physical integrity of the bottles, using them as a raw material for new direct reuse and repurposing applications. Tensile tests were conducted on samples cut from polyethylene terephthalate (PET) bottles considering different sets of variables: cutting method, orientation of the samples and degradation method. The results clearly demonstrate the superior strength characteristics, and minimal effect of degradation, showing the potential for a whole new field of applications where these recovered materials could be used. However, successful adoption of this new concept relies on structural changes to the operations of recycling and manufacturing companies as well as legislators, regulators and the general public.
\end{abstract}

\section{Introduction}

The current human period of history has already been referred to as the plastic age (Cozar et al., 2014). According to PlasticsEurope (PlasticsEurope, 2016), in

\footnotetext{
1 School of Biosystems and Food Engineering, University College Dublin, Belfield, Dublin 4, Ireland irene.villafane@ucdconnect.ie; tom.curran@ucd.ie

${ }^{2}$ School of Mechanical and Materials Engineering, University College Dublin, Belfield, Dublin 4, Ireland; colin.keogh@ucd.ie

${ }^{3}$ School of Biomolecular and Biomedical Science, University College Dublin, Belfield, Dublin 4, Ireland; emmanuel.reynaud@ucd.ie
} 
204 Assessment of the mechanical properties of pet polymer material from recovered plastic bottles

2015, the global production of plastic resins has reached 322 MMT (million metric tonnes), a $690 \%$ increase since 1975 . Almost $40 \%$ were used in the packaging industry (PlasticsEurope, 2016), in other words, for a single use. The amount of plastic materials produced, used and disposed from 1950 to 2015 (Geyer et al., 2017), accounts for the global primary production of polymer resins, synthetic fibres and additives [8,300 MMT], in-use stocks [2,500 MMT and 100 MMT (primary and secondary respectively)]. Then 60\% (4,900 MMT) are discarded to landfills, 10\% (800 MMT) incinerated (with or without energy recovery) while only $7 \%(600 \mathrm{MMT})$ is recycled.

Even though in recent years the rate of recycled plastic has risen to $29.7 \%$ (data from Europe in 2014) (PlasticsEurope, 2016), the methods of disposing and recycling plastic waste materials are still inefficient. In addition, the increased consumption, rapid disposal of plastic materials and high stability of plastic products leads to the accumulation of plastic waste in the environment. This is a global problem affecting all areas of the planet, with most of the impact exhibited in marine and coastal environments. The superior characteristics which makes plastic attractive for use such as low density and high durability are a big disadvantage once the material is in the environment. Plastic objects can last hundreds of years in the natural environment (Gregory et al., 2003) and that makes them a significant threat to marine ecosystems (Eerkes-Medrano et al., 2015; Ivleva et al., 2017; a Costa et al., 2016). Due to these recurring issues, current marine plastic quantities and the influx of further quantities, it has become one of our society's major environmental problem (Jambeck et al., 2015). It was demonstrated that between 4.8 and 12.7 MMT of plastic waste (estimated to be equivalent to $500,000,000,000$ plastic drink bottles) from 192 coastal countries around the globe entered the marine environment in 2010 alone.

The majority of these bottles are thin walled plastic bottles, made from PET (polyethylene terephthalate) or HDPE (high density polyethylene). This work focuses on PET bottles, due to their prevalence in consumer sales, and resultant occurrence in the natural environment. Thin-walled plastic bottles are manufactured by using an injection stretch-blow moulding (ISBM) process. The mechanical properties of the material after the manufacturing process, such as the chain orientation of the polymer molecules, depends on many factors and operating conditions. The orientation pattern in PET bottles is complex because of the nature of biaxial orientation that varies along the bottle surface (Awaja et al., 2005). However, some studies (Billon et al., 2014) determined that the orientation of the chains is variable along the bottle axis (Fig. 1).

Once the plastic bottles reach the end of their originally designed functional life, they are either recollected and recycled or directly disposed of. The collection is done either via curbside collections or deposit systems (Welle, 2011). Bottles are 
then cleaned and sorted out in the different materials: plastic used in the main body of the bottle (usually PET or HDPE), cap and neck ring (usually PP (polypropylene) or HDPE), label (made from plastic (LDPE, low density polyethylene) or paper and ink) and the glue used to hold the label. Normally, plastic bottles are segregated from mixed waste collections, then broken apart (with mechanical or chemical systems) either before or after the sorting of materials to create flakes which will be used for different applications such as fibres and moulding new bottles (Noone, 2008). In many cases these resulting flakes are separated again with different methods such as density separation or air flow separation. This process has a low efficiency overall and a high energy consumption due to the complex machinery required to successfully complete these separations and the transport required to translate the recollected plastic waste between the different stages of its life cycle. In addition, more energy is required in the shredding process itself. This energy consumption and its resulting $\mathrm{CO}_{2}$ footprint increase could be reduced by preserving the physical integrity of the products avoiding many separation, manufacturing and transport stages, and therefore, retaining of their initial mechanical properties at lower energy/ $/ \mathrm{CO}_{2}$ emission costs.

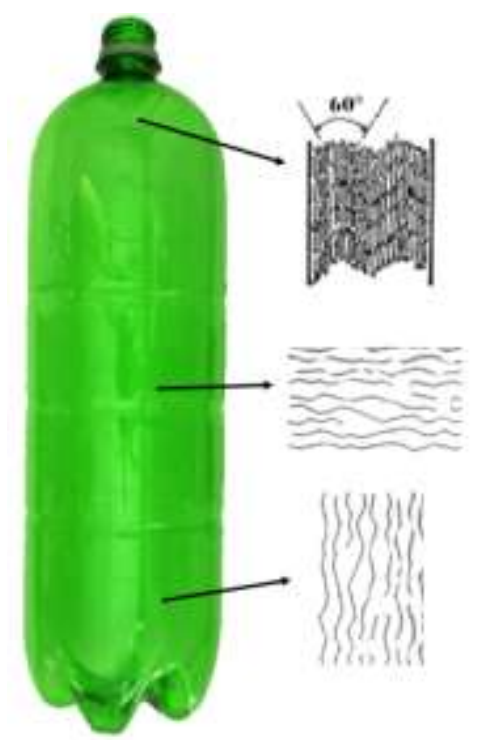

Figure 1. Chain orientation in the PET bottles. Modified from (Billon et al., 2014)

Even though most of the plastic packaging waste is collected and processed, there are alternatives based on the preservation of its physical integrity, in this case, recovered complete plastic bottles. One of those alternatives, for example is a 
'Plastic Bottle Village' constructed in Panama (Plastic Bottle Village, 2017). In which a complete two floor house was constructed from complete PET plastic bottles. Other similar initiatives such as 'Plastiki' constructed a boat using 12,500 2-litre complete plastic bottles and sailed 8,000 nautical miles from San Francisco to Sydney (The Plastiki, 2017). In addition to this many DIY and craft applications such as plastic boxes made out of the body sheet of the bottles (Fig. 2) can be found online which allows the production of a variety of objects including furniture, tools and toys. All utilizing complete or sections of plastic bottles as raw materials rather than flakes.

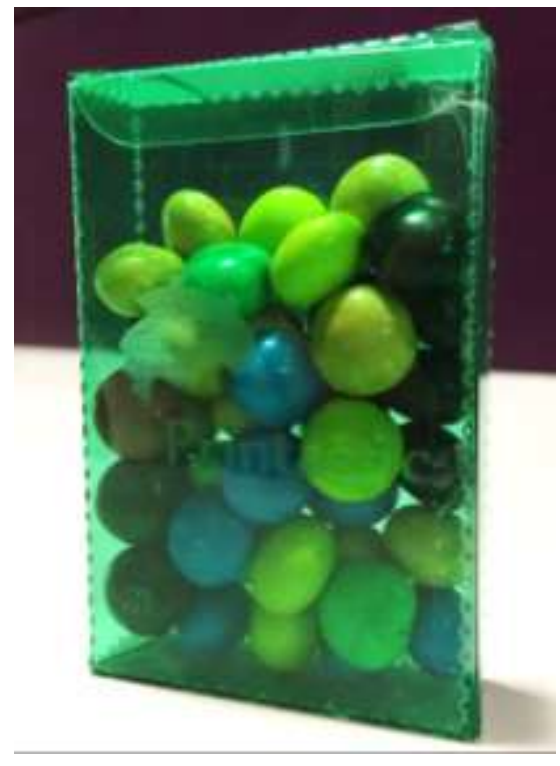

Figure 2. Plastic box made from the body sheet of a bottle.

This study aims to assess the mechanical properties of recovered PET sheets to understand the potential of these recovered PET materials (in a non-shredded form) for further specific uses. These uses will be integrated in a new recovering and recycling plastic waste system based on the preservation of the physical integrity of the recovered plastic materials. Doing this, the energy consumption of the remanufacturing stages (separation, shredding and re-melting processes) will be reduced or even avoided altogether. The assessment of the material properties will be done by carrying out numerous tensile tests considering different sets of variables. 


\section{Materials and Methods}

\subsection{Testing Standards}

Mechanical testing of recovered plastic material is quite novel, with very little previous information available, resulting in there being no reference for the methods of testing the materials. To perform the tests the ISO standard 'ISO 527-31995: Plastics - Determination of tensile properties - Part 3: Test conditions for films and sheets' was used to guide the testing.

\subsection{Sample Preparation}

The procedure of creating the specimens consisted of the collection of a standardized commercially available plastic bottle (green PET bottle, 2 litres

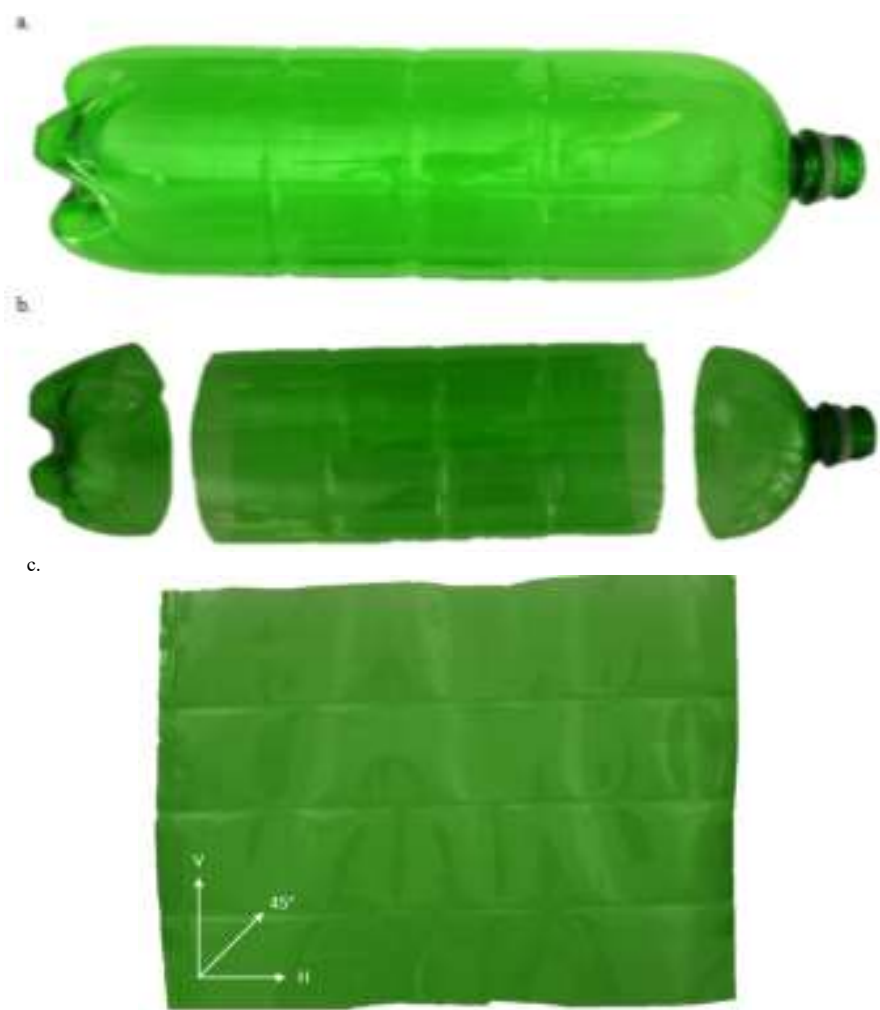

Figure 3. a. Complete Bottle, b. Top and Bottom Removed and c. Curled Flat Sheet

capacity, no corrugations as seen in Fig. 3.a), removal of the top (from the shoulder) and the bottom from the bottle (Fig. 3.b), resulting in a curled flat sheet (with size slightly smaller than A4 paper sheet) consisting of the main body of the 
bottle (Fig. 3.c). Once the plastic sheets were collected, the specimens were cut considering different scenarios, presented in Tab. 1. Every combination of variables was considered and a minimum number of five specimens per combination was cut.

Tab. 1. Testing scenarios

\begin{tabular}{|c|c|c|}
\hline & & Parameters \\
\hline \multirow{2}{*}{$\begin{array}{l}\text { Cutting } \\
\text { Method }\end{array}$} & \multicolumn{2}{|l|}{ Laser (L) } \\
\hline & \multicolumn{2}{|l|}{ Guillotine $(\mathrm{G})$} \\
\hline \multirow{3}{*}{ Orientation } & \multicolumn{2}{|l|}{ Vertical (V) } \\
\hline & \multicolumn{2}{|l|}{ Horizontal (H) } \\
\hline & \multicolumn{2}{|l|}{$45^{\circ}\left(45^{\circ}\right)$} \\
\hline \multirow{5}{*}{$\begin{array}{c}\text { Degradation } \\
\text { Method }\end{array}$} & \multicolumn{2}{|l|}{ UV light (1 week) } \\
\hline & \multirow{2}{*}{ Water degradation } & Sea water ( 2 weeks $)$ \\
\hline & & Fresh water (2 weeks) \\
\hline & \multirow{2}{*}{ Thermal degradation } & High temperatures $\left(50^{\circ} \mathrm{C}, 6 \mathrm{~h}\right)$ \\
\hline & & Sub-zero temperatures $\left(-18^{\circ} \mathrm{C}, 24 \mathrm{~h}\right)$ \\
\hline
\end{tabular}

The cutting method refers to the method used to cut the samples from the plastic sheet. Laser cut specimens suffered a minimal thermal treatment on the edges of the sample which resulted in some hardening of the material (Fig. 4). As mentioned in the introduction, the orientation of the PET chains differs depending

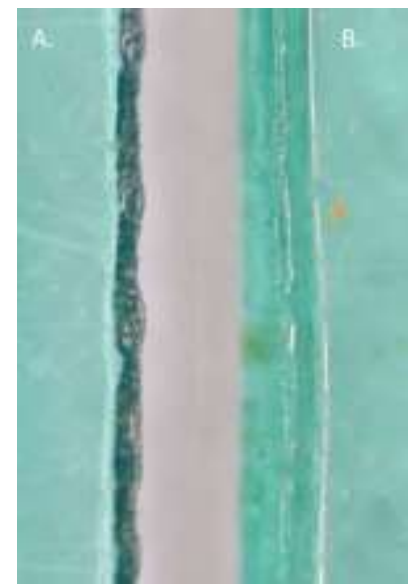

Figure 4. Cutting method comparison: a. guillotine cut (left) vs. b. laser cut (right) 
on the parts of the bottle from which they originate and therefore influences the mechanical properties of the material. Due to this and in order to have representative results from the tests, the three main orientations were considered (horizontal, vertical and $45^{\circ}$, Fig. 2.c). The vertical samples are those cut along the main axis of the bottle. Five different degradation methods were considered. They represent the conditions of a plastic bottle collected from the environment: water degradation (from fresh water and marine sources), thermal degradation (at subzero and raised temperatures) and UV light degradation.

\subsection{Testing Methods}

Houndsfield H20K-W and Lloyd LR30 K Tensile Test machines were used for the testing of the samples, with the testing speed selected according to ISO 527$3-1995$ (selected testing speed $=50 \mathrm{~mm} / \mathrm{min}$ ). The thickness and width of the samples were measured before the tests and all the specimen details were recorded.

\section{Results}

Samples cut from plastic sheet recovered from complete plastic bottles were tested for their mechanical properties. The stress of the average breaking point were $210 \mathrm{MPa}$ and $140 \mathrm{MPa}$, for the laser and guillotine cut sets respectively (Fig. 5). Those initial results show the exceptional characteristics of the recovered PET

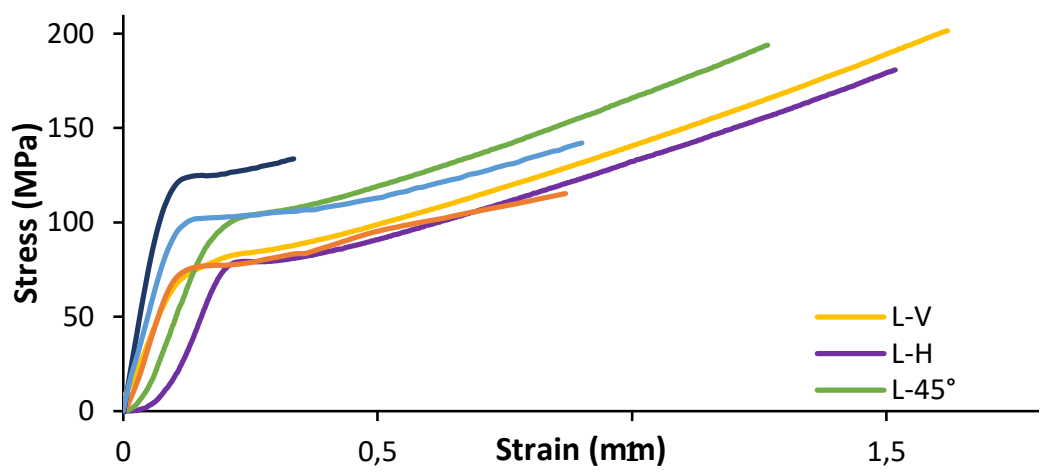

Figure 5. Average stress-strain results of PET Reference Tests

plastic sheet material. These values can be compared to the ones listed in materials data books (CUE, 2003) in which the maximum tensile strength of PET is between 48.3 and $72.4 \mathrm{MPa}$. Other studies (Cinelli et al., 2016) report PET strength at 150 $\mathrm{MPa}$. The cutting method as well as the orientation of the samples influence their tensile strength (Fig. 5). Laser cut samples supported higher stress and strain at the 
breaking point, with approximately $33 \%$ more stress and $45 \%$ more strain. On the other hand, the impact of the orientation was higher in guillotine cut samples. Horizontally cut samples had the molecular chains of the polymer aligned in a different orientation than the load. This implies that only a very short elongation was possible before the breaking point. Still, the strength supported by the samples was just as high or higher (approximately 5\%) than the vertically orientated ones.

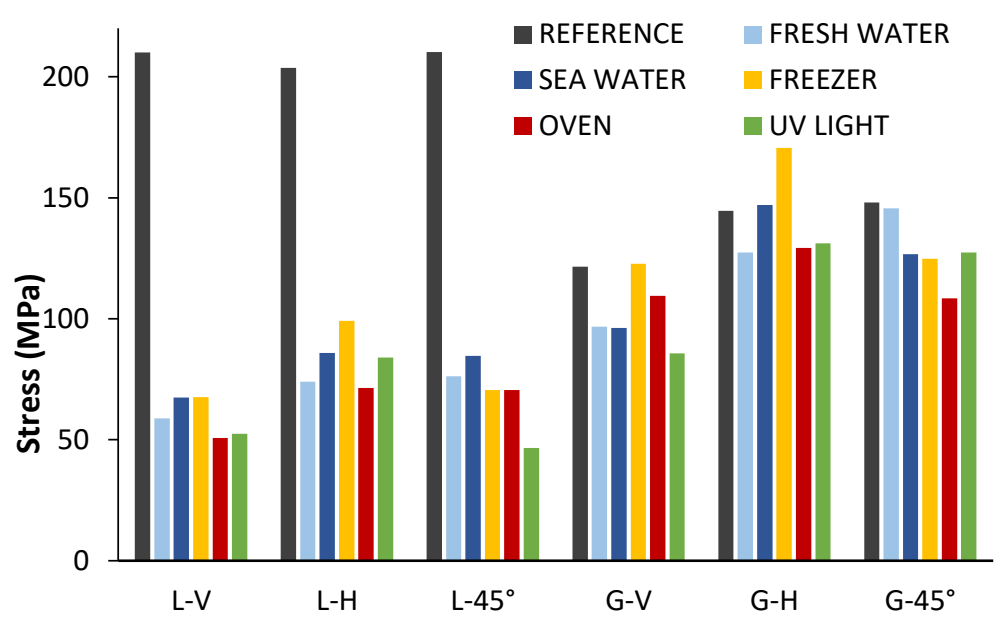

Figure 6. Average stress of the breaking point comparison

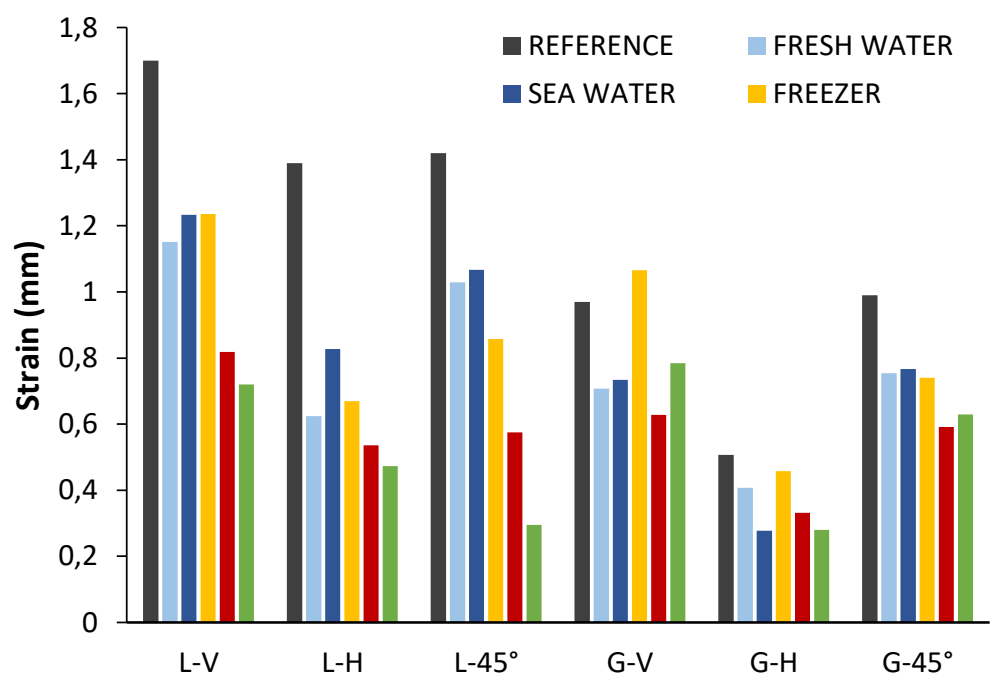

Figure 7. Average strain of the breaking point comparison 
The degradation only affected the polymer tensile strength at a very low rate, but it had a small impact on the final strength of the samples. The most effective degrading method was UV light exposure, followed by raised temperature degradation (Figs. 6 and 7). Sub-zero temperatures and water degradation (fresh or marine) had minimal effects on the measured strains/stresses (Figs. 6 and 7). In the comparison of the stress of the breaking point (Fig. 6), the main result is the effect of the cutting method versus the degradation. Laser cut samples when degraded, seemed to lose most of their mechanical strength approximately $65 \%$ compared to the reference ones, and 56\% compared to the guillotine cut ones. Guillotine cut samples lost only approximately $10 \%$ of stress compared to the reference tests. The differences in the strain of the breaking point (Fig. 7) are also higher for laser cut samples, $40-60 \%$, than for guillotine cut ones, $10-30 \%$.

\section{Discussion}

The main aim of this study was to analyse the mechanical properties of the plastic sheets from recovered complete single use PET bottles. These excellent properties illustrate the potential of a new recycling system in which the recovered plastic material is reused and repurposed preserving part of its initial physical integrity. This system could replace the actual system based on the physical size reduction of the recovered plastic as well as reducing energy consumption of waste management companies and $\mathrm{CO}_{2}$ emissions.

In addition to the mechanical assessment of the material, the influence of variables such as cutting method, orientation and degradation method was tested. The laser cutting process samples caused a thermal effect on the edges of the samples. The heat modified edges made the samples stronger in the reference tests. However, after being degraded, every laser cut sample was weaker than the reference and the guillotine cut ones. The reason for this could be that any kind of degradation affects the sample edges more because they are already altered. The guillotine cut samples, on the other hand showed a minimal loss of strength which proves the outstanding characteristics of the plastic even when it is collected from the environment but also shows that consideration of the material must be taken into account when selecting the correct reprocessing method.

However, in order to achieve this aim of reusing and repurposing PET or any other plastic materials, some societal changes need to be made. The collection of plastic bottles could be standardised and facilitated by both manufacturing companies and waste/recycling companies. Mixing recyclable waste complicates the reprocessing of plastic as it is contaminated by other waste types. Nevertheless, the major changes which are needed must occur in the plastic bottle manufacturing process. Most plastic bottles consist of a mix of at least four different materials (e.g. PET, HDPE, paper/Vinyl...) which makes the reprocessing of plastic bottles 
extremely difficult as the glue and the neck ring are susceptible of being very difficult to efficiently remove, either by hand or in larger scale mechanical separation systems. In addition, a standardised shape of the body and the neck of the bottles would facilitate their improved identification, recollection and reprocessing. Nowadays, plastic waste is not recycled to its maximum ability. The plastic waste collected in some European countries, such as Ireland, is in most cases mixed, baled and compressed with other recyclables, causing the sorting of waste by material type almost impossible. The fate of recyclable wastes lies in export to developing countries such as China or incineration with other municipal solid wastes (with or without energy recovery). The energy recovery rates with the incineration of plastic waste are very low when compared to the energy required to create a new plastic object. This is an inefficient waste of raw materials and energy, taking into account the outstanding properties that the material has proven to have when recovered from waste streams.

Changing this situation should be one of the main objectives of our society. The whole system of the life cycle assessment of plastic packaging materials needs to be properly evaluated, to allow the change and improvement of future waste collection systems. New manufacturing and recollection systems supported by appropriate and well-informed legislation needs to be established. The authors of this study propose solutions for this at a governmental, domestic, local and community level. This will allow participants to more easily deal with their own recyclable plastic waste while they benefit financially, ethically and environmentally during the process.

This study can be used as a basis and proof-of-concept for further and deeper research. It has been proven that the plastic material used in packaging objects has outstanding characteristics when recovered and more materials: HDPE, PE, etc. can be analysed for similar evaluation. Additional investigations into the influence of other variables such as texturing, shaping or colouring of the plastic can be undertaken. More varied and longer degradation periods are also proposed for a more comprehensive study; in most of the studies referenced the degradation periods in water or UV light lasted months.

\section{Conclusions}

The aim of this project was to assess the mechanical properties of the plastic material used in recovered PET plastic bottles, with the objective of proving the feasibility of using them without destroying their physical integrity for reuse purposes. The mechanical strength of the non-degraded samples was found to be outstanding in terms of strain-stress supported regardless of the cutting method or the orientation considered. Furthermore, the degradation of the plastic material 
resulted in only a slight loss of strength. This loss was higher in the laser cut samples, those which had suffered a thermal alteration on the edges. For guillotine cut samples the differences between degraded and non-degraded samples were negligible.

In addition to the main result, the influence of each variable considered was also studied. The cutting method made laser cut samples stronger when the material was not degraded and weaker when it was. On the other hand, guillotine cut samples did not suffer almost any degradation or further mechanical loss. The orientation affected the results mostly in terms of the strain or elongation supported by the samples. While the stress or the breaking point did not present a high variation between the different orientations, the strain suffered by the vertically cut samples was in some cases four times higher than the ones suffered by the horizontally cut ones. The different degradation methods influenced the results mostly for the laser cut samples. The method which caused most degradation was UV light exposure and therefore a longer degradation period is proposed. Thermal and water degradation methods presented very low degradation effects. Theses result highlight the potential for direct reuse of waste plastic materials, while giving the possibility to simplify the waste collection system to preserve the physical integrity of the material thus increase the potential mechanical performance and thus the value.

\section{References}

Awaja, F., Pavel, D. (2005) Recycling of PET. EUROPEAN POLYMER JOURNAL 41(7), 1453-77.

Billon, N., Picard, M., Gorlier, E. (2014). Stretch blow moulding of PET; structure development and constitutive model. International Journal of Material Forming. 7(3), 369-78. (10.1007/s12289-013-1131-1)

Cinelli, Patrizia, Schmid, M., Bugnicourt, Elodie, Coltelli, Maria, Lazzeri, A. (2016) Recyclability of PET/WPI/PE Multilayer Films by Removal of Whey Protein IsolateBased Coatings with Enzymatic Detergents. Materials 9(6), 473.

da Costa, J.P., Santos, Patrizia S.M., Duarte, A.C., Rocha-Santos, Teresa. (2016) (Nano)plastics in the environment - Sources, fates and effects. Science of the Total Environment 566-567, 15-26

Cózar, A., Echevarría, F., González-Gordillo, J.I., Irigoien, X., Úbeda, Bárbara., Hernández-León, S., Palma, A.T., Navarro, Sandra, García-de-Lomas, J., Ruiz, Andrea. et al. (2014). Plastic debris in the open ocean. Proceedings of the National Academy of Sciences of the United States of America 111(28), 10239-44.

Department CUE. (2003) Materials Data Book 
214 Assessment of the mechanical properties of pet polymer material from recovered plastic bottles

Eerkes-Medrano, D., Thompson, R.C., Aldridge, D.C. (2015) Microplastics in freshwater systems: A review of the emerging threats, identification of knowledge gaps and prioritisation of research needs. WATER RESEARCH 75, 63-82

Geyer, R., Jambeck, Jenna R., Law, Kara L. (2017) Production, use, and fate of all plastics ever made. Science Advances. 3(7)

Gregory, M.R., Andrady, A.L. (2003) Plastics in the marine environment. Plastics and the Environment, 379-401.

International Standard (ISO). (1995) International Standard (ISO): PlasticDetermination of tensile properties - Part 3: Tensile conditions for films and sheets. ISO 527-3-1995.

Ivleva, Natalia P., Wiesheu, Alexandra C., Niessner, R. (2017). Microplastic in Aquatic Ecosystems. Angewandte Chemie International Edition 56(7), 1720-39.

Jambeck, Jenna R., Geyer, R., Wilcox, C., Siegler, T.R., Perryman, M., Andrady, A., Narayan, R., Law, Kara L. (2015) Plastic waste inputs from land into the ocean. SCIENCE. 347(6223), 768-71. (10.1126/science.1260352)

Noone, A. (2008) Collected PET bottles. Proceedings of 13th International Polyester Recycling Forum

Plastic Bottle Village. (2017) [Available from: www.plasticbottlevillage.com]

PlasticsEurope. (2016) Plastics - the Facts 2016. Brussels, Belgium [Available from: http://www.plasticseurope.org/Document/plastics---the-facts-201615787.aspx?FolID=2]

The Plastiki. (2017) [Available from: http://theplastiki.com]

Welle, F. (2011). Twenty years of PET bottle to bottle recycling-An overview. 2011. Resources, Conservation \& Recycling. 55(11), 865-75. 